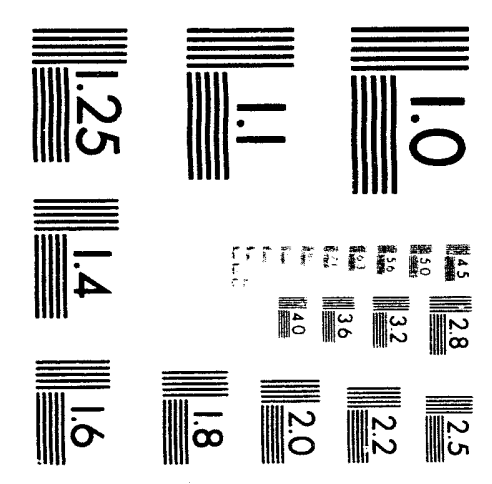



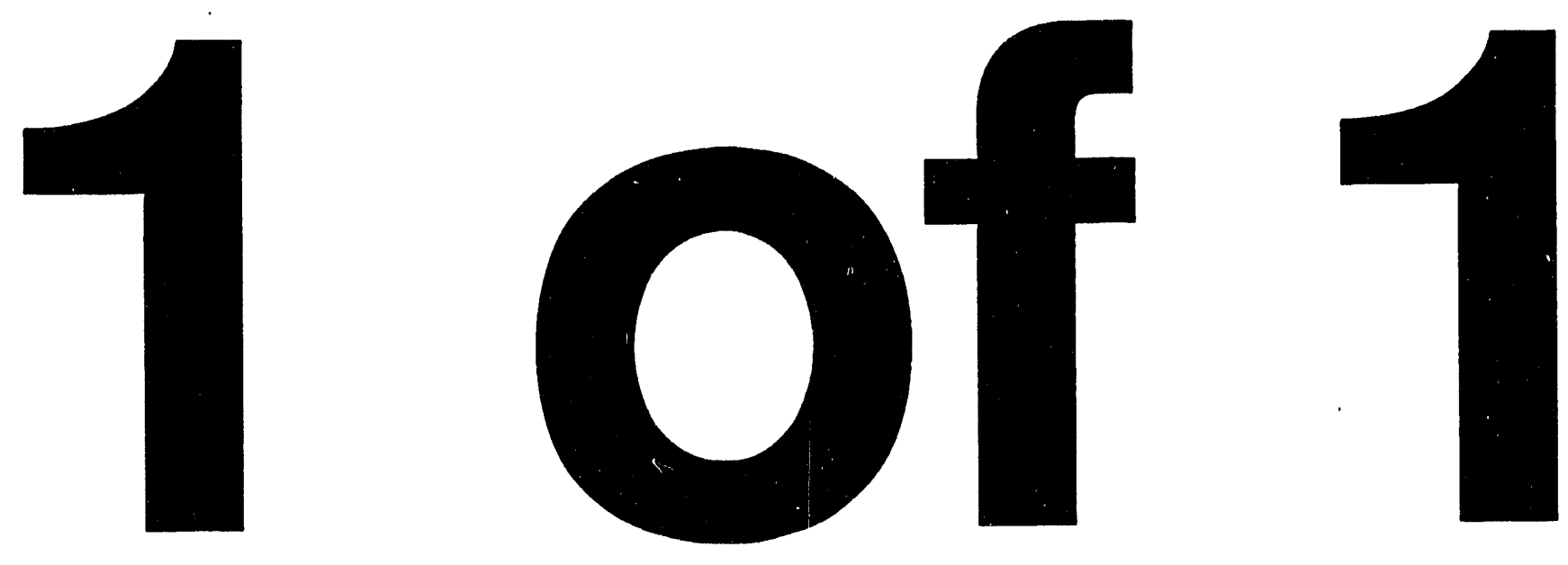
GA-A21427

\title{
NEW THOMSON SCATTERING LASER CONTROL FOR DIII-D
}

\author{
by \\ C.C. MAKARIOU, R.E. STOCKDALE, T.N. CARLSTROM, \\ C.-L. HSIEH, and G. BRAMSON
}

This is a preprint of a paper to be presented at the 15th IEEE/NPSS Symposium on Fusion Engineering, October 11-15, 1993, in Hyannis, Massachusetts, and to be printed in the Proceedings.

Work supported by

U.S. Department of Energy

Contract DE-AC03-89ER51114

GENERAL ATOMICS PROJECT 3466

OCTOBER 1993

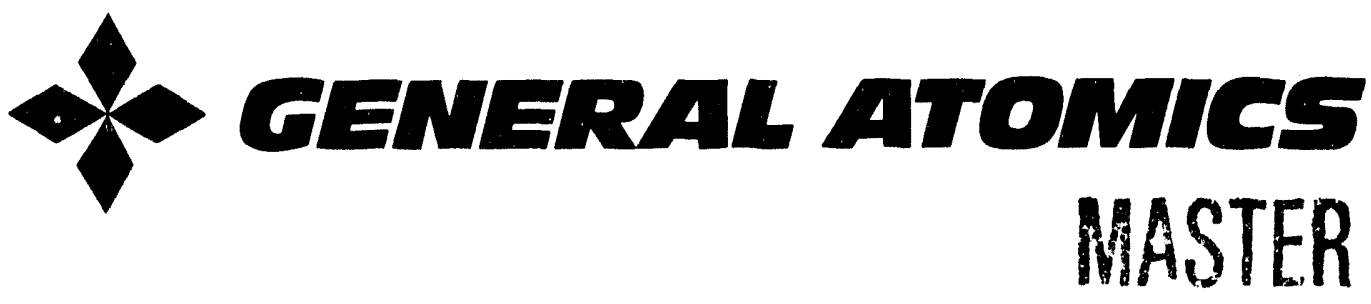




\title{
NEW THOMSON SCATTERING LASER CONTROL FOR DIII-D*
}

\author{
C.C. Makariou, R.E. Stockdale, T.N. Carlstrom, C.-L. Hsieh, and G. Bramson \\ General Atomics \\ P.0. Box 85608, San Diego, California 92186-9784
}

\begin{abstract}
A Laser Control system has been built for the DIII-D Thomson Scattering Diagnostic $[1,2]$. This new system has provided the capability to place the laser probe pulses with one microsecond timing precision throughout the DIII-D shot. The new system fires the $\mathrm{e}_{\mathrm{g}} \mathrm{ht}$ lasers with a programmable sequence which repeats every $50 \mathrm{~ms}$. If one wants to probe the plasma at a higher rate to study a fast paced event, the new control circuit can fire all charged lasers in rapid succession (BURST MODE). This burst rate is programmable. The new Laser Control system successfully replaced the previous control scheme which consisted of three VME Motorola $68030 \mathrm{com}$ puters (one host under UNIX VME V/68 and two interrupt driven targets under VME Exec. The old system was not successful due to the many VME interrupts needed to service the lasers. The new hardware approach is much more reliable. The old system still controls data acquisition and as a monitoring system since it does not have the burden of controlling the lasers. A brief description of the Thomson Scattering diagnostic will be presented with emphasis in the new upgraded laser firing control system and data acquisition timing control.
\end{abstract}

\section{INTRODUCTION}

The Thomson scattering system on the DIII-D Tokamak has eight $20-\mathrm{Hz} \mathrm{Nd}$ :YAG lasers. Multiple lasers provide capability for higher overall repetition rate and burst mode capability where individual lasers are fired in rapid succession, limited only by the data acquisition rate $(100 \mu$ s per laser pulse). Several signals control the charging and firing of each laser. These are: charge command, fire command, and lasing enable. Each laser sends a ready signal before charge commands can be accepted. The laser responds with "end of charge" when ready to accept a fire command. This sequence must be maintained accurately and consistently to avoid problems with laser operation. Each laser's capacitor bank needs approximately $20 \mathrm{~ms}$ to charge. The lasers are designed to operate at $20 \mathrm{~Hz}$. Due to the complexity of the system and the plethora of control signals for each laser a hardware control system was necessary to control the firing operation.

\section{SYSTEM OVERVIEW}

The Thomson Scattering diagnostic consists of: eight lasers, an electronics cage, a fiber optic interface cage, seven
CAMAC crates, two VME crates, a MicroVAX 3400, and a programmable logic controller (Fig 1). Two of the CAMAC crates contain the Background and Scattered light FERA 4300 aquisition modules, and the other five contain input and output interface modules, timing sequencers, gate timers, data loggers, time stamping modules, and the burst generator. One VME crate contains two ECL interfaced RAM modules made by Creative Electronics Systems (CES) that collect data from the FERA crates and the other contains the target data acquisition processor and two Eltec interrupt generator modules. The MicroVAX 3400 is used to program all the modules in the crates that control the firing of the lasers. The MicroVAX also communicates with the VME data acquisition computer and performs data archival. The programmable logic controller maintains safe operation of the lasers.

The Thomson control circuit utilizes the flexibility that the Jorway-221 offers both in timing accuracy and programmability. The Jorway-221 is a 12 channel digital sequence module with $2 \mathrm{~K}$ memory and a 24 bit time counter. A specially modified Jorway-221 controls the charging sequence for the eight DIII-D lasers: It is able to reset in the middle of a sequence with an external roll-back pulse and wait for the next start pulse to start running again. This feature gives us capability to synchronize the firing operation with the DIII-D plasma shot. The start pulses are produced from a 16 bit programmable counter that counts 50,000 cycles from the DIII-D master clock. This counter can also be synchronized with the DIII-D synchronization pulse that arrives nineteen seconds before each plasma shot.

The charging sequence is passed to the control circuit which is housed in a VME format cage with a custom back plane that can carry 84 signals on P1 and 84 signals on P2 conncetors across the bus.

The upper bus has eight identical circuits on four $3 \mathrm{U}$, 0.6 -in. wide cards that are dedicated to control the charging and to keep track of each laser's end of charge signal. Each circuit has a 16 bit selectable delay counter and counts 47,000 cycles from the $1 \mathrm{MHz}$ DIII-D master clock. Fig. 2 shows a basic block diagram of the delay/counter circuit. If the "End of charge" flag is set the circuit sends a "regular" fire request on the bus. The delay is used to provide the burst mode circuit a time window in which a laser's capacitor bank remains charged $(30 \mathrm{~ms})$ and in which a burst trigger can be accepted.

A specially designed burst sequencer card module monitors the "End of charge" flags for each laser. It checks the eight End of charge flags for a charged laser and points at the

*Manuscript received October 11, 1993. This work was supported by the U.S. Department of Energy under Contract No. DE-AC03-89ER51114. 


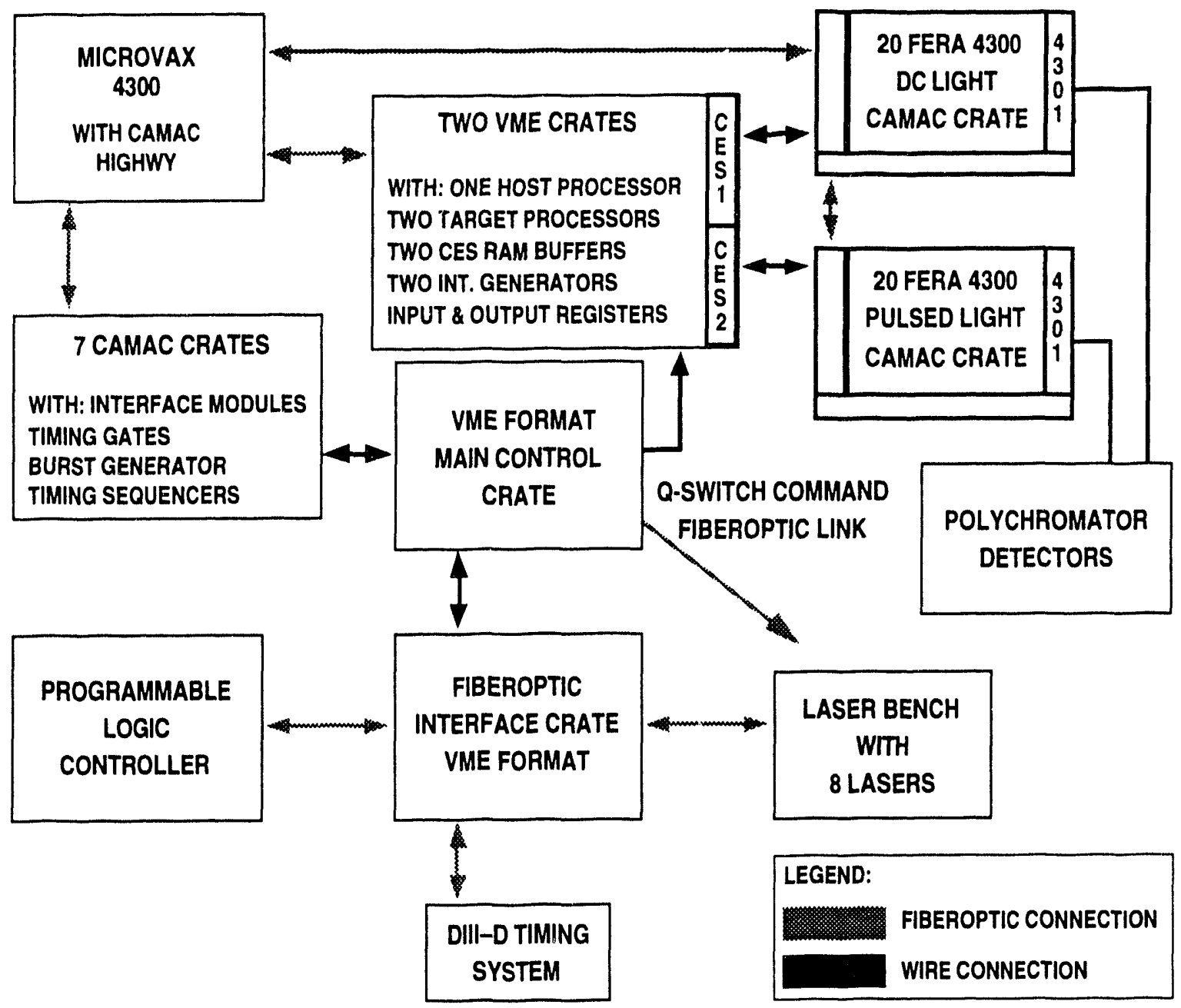

Fig. 1. Thomson system hardware map.

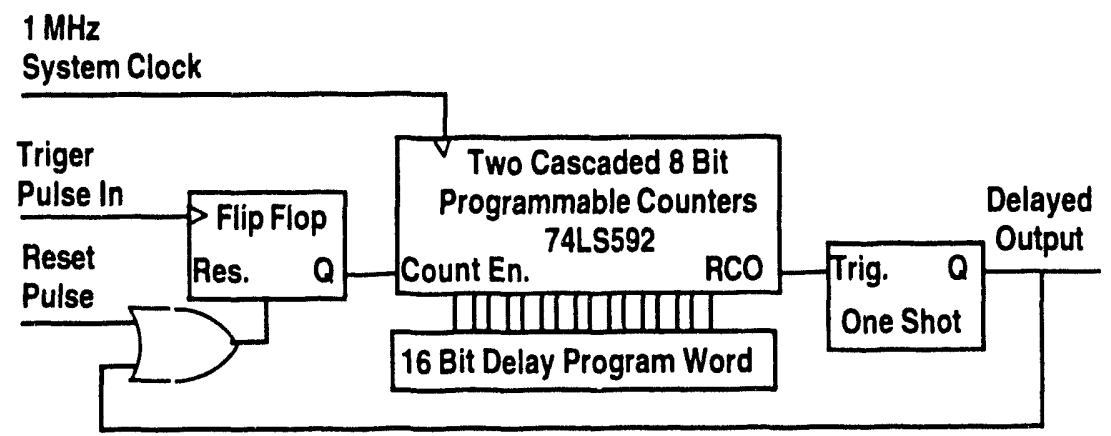

Fig. 2. Microsecond precison delay block.

least recently charged laser. In the event of a burst request a pulse train is produced by the burst generator (a custom CAMAC module). This module can produce three separate sequences of one to eight pulses with preprogrammed intervals. The burst generator is triggered from event detectors that are looking for transient events of interest in the plasma. The burst sequencer accepts the pulse train and directs each pulse to an available (charged) laser. The burst capability is at least five of eight lasers at all times (if the lasers are charged evenly through the cycle) or up to eight if all are charged at the same time.

On the lower bus are eight identical circuits on four $3 \mathrm{U}$, 0.6-in. cards that flash the laser and Q-switch the cavity at the detection of a fire request from the circuit described above. 
The lasers require a period of about $250 \mu$ s to achieve population inversion. For optimum performance this time is slightly different for each laser. The circuit for each laser has two nine-bit digitally-set delays (1-512 $\mu \mathrm{s})$. One delay is used to delay the flashing command and the other is used to delay the Q-switching time from the fire request signal. The Q-switch delay is set for all eight lasers to be the same and the flashing delay (latency) is set for optimum lasing power for each individual laser.

The Q-switch requests are routed through the bus to another board 6U, 1.2-in. wide. The Q-switching board is responsible for sending a Q-switch command to each laser's cavity pockel cell via eight separate fiber optic links. If two or more Q-switch commands signals arrive on this board on the same microsecond the first edge produces a pulse which is slightly delayed. This pulse is passed to eight half-nanosecond step 8-bit programmable delay chips (PDU 256-.5) and the Qswitch request for each laser is used as an enable for the appropriate delay chip. The result is that a group of Q-switch pulses are issued from this card with an adjustable relative time difference from a single time reference pulse (Fig. 3). The Q-switching delay of each laser is appropriately adjusted so the laser pulse arrives at the same time on the target. In this way the eight lasers can be fired simultaneously as one combined laser (Group Mode.)

Specially designed cards are used to carry all the signals from the bus to the outside world as shown in Fig. 4. These cards are $6 \mathrm{U}$ and have $\mathrm{P} 1$ and $\mathrm{P} 2$ connectors on the VME bus side and four external connectors that can carry 32 signals each. The PCB trace from each connector pin ends with a speedy-wire pin on the board. Input and output connections to the card are easily made by linking the appropriate pins with wire-wrap wire.

To avoid ground loops and noise problems, fiber optic cables are used to interface the control circuit to the programmable logic controller and the lasers, and to accept commands from the DIII-D control system. Another VME cage is used for the fiber optic interfaces. The fiber-optic interface cage has ten 1.2-in. spaced slots filled with custom made fiber-optic transmitter/receiver $6 \mathrm{U}$ cards and four 0.6 -in. spaced slots which hold the interconnect cards. Two interconnect cards are used to connect the appropriate lines from the fiber optic sage to the control circuit cage. Two more interconnect boards are used in the control cage to connect output and input CAMAC hased control registers, miniature coaxxial connector points for easy access to the bus, and the burst clock generator module.

The data acquisition trigger control circuit produces a precisely delayed pulse to trigger the Lecroy 4300 FERA Digitizer modules. The acquisition system has to wait for the scattered light to return from the DIII-D machine and start acquiring data when the detected signal is within a 65 ns gate. It also acquires data for background light intensity $100 \mathrm{~ns}$ prior to the arrival of the scattered light Fig. 5.

Two gate pulses are generated after each laser pulse. The position in time of the two gate pulses relative to the detected pulse has to be precisely adjusted to achieve maximum scat-

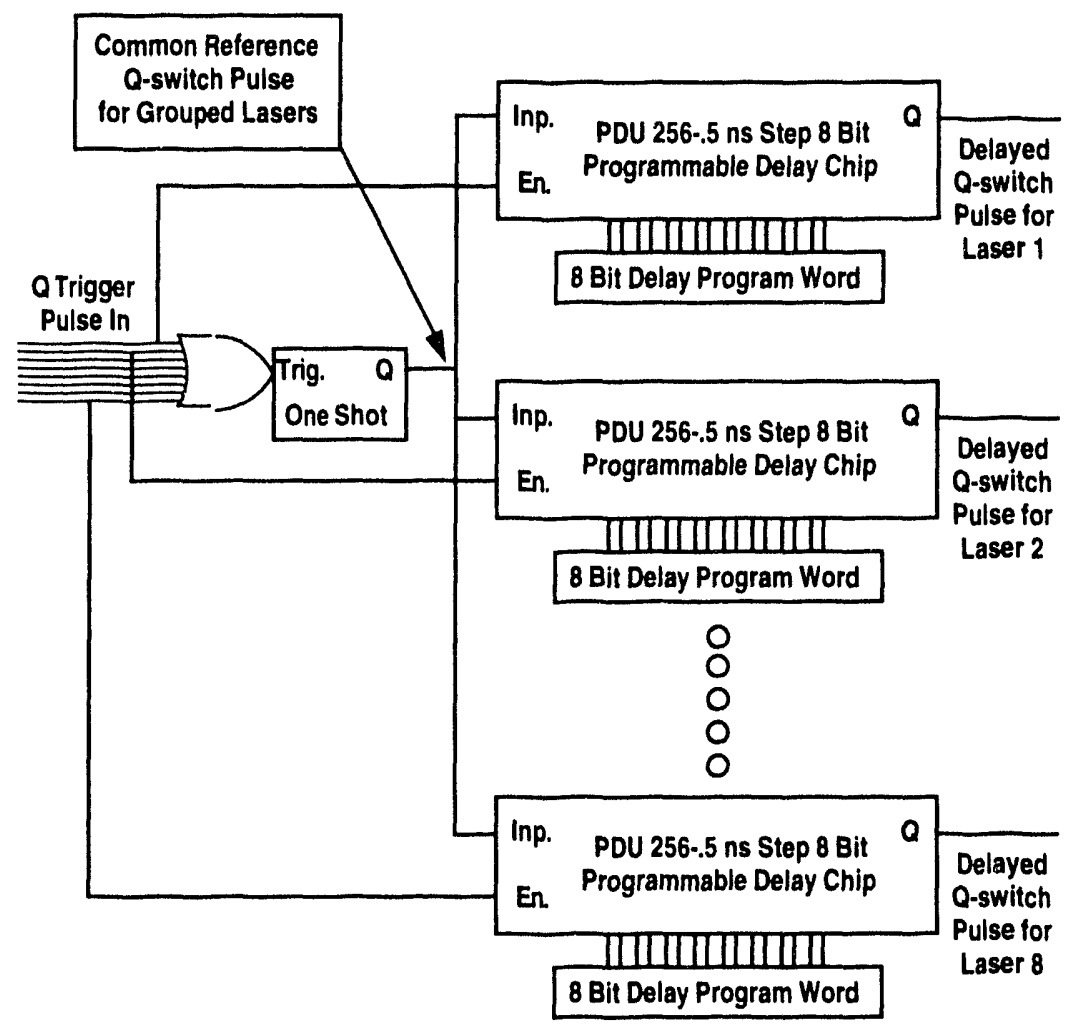

Fig. 3. Q-switching synchronization/delay circuit for group firing. 


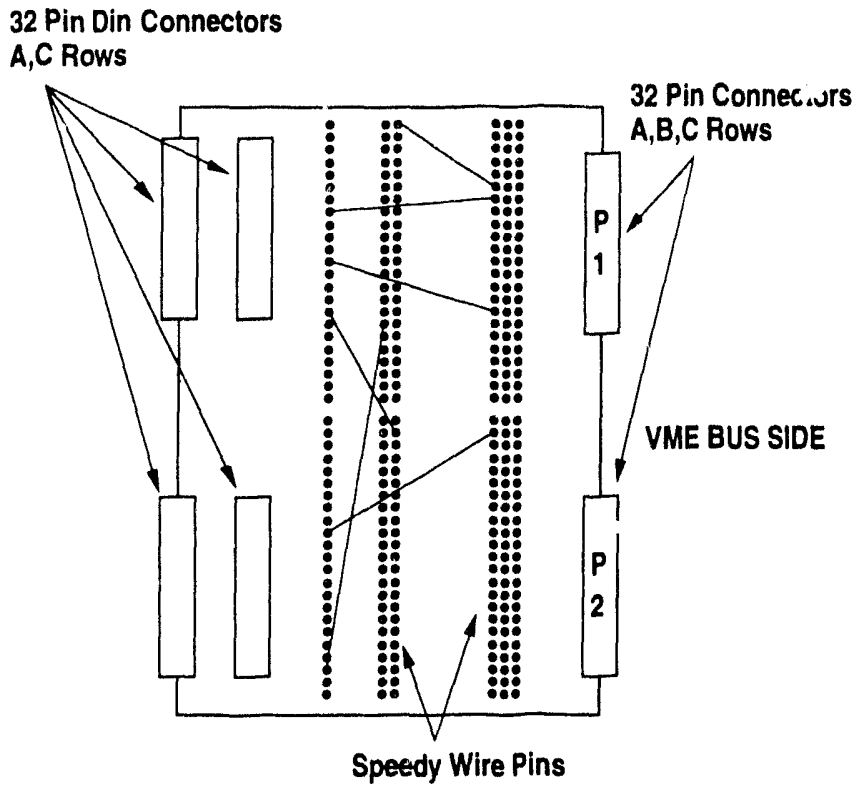

Fig. 4. Interconnect VME format patch panel.

tered signal to background light ratio. To achieve this a PDU 256-0.5 digital programmable delay chip was used to delay the light pulse received from an integrating sphere located near the lasers. The trigger control circuit is used in conjunction with a Jorway-221 programmable sequence module to provide scan capability. The Jorway-221 is programmed to produce an ascending sequence of eight digital bits which increments by one after a fixed number of laser pulses. The eight bits feed the PDU 256-0.5 control inputs. This produces a data stream where the collection gate moves in time relative to the arriving light pulse in $0.5 \mathrm{~ns}$ steps within a predetermined usefull range. The data are plotted and an optimum delay is chosen. The eight bits are then permanently selected by a DIP switch located on the board. The programmable (half nanosecond precision) delay generator with scan capability was a very powerful feature added to the data acquisition side of the control system. This scan capability provides a fast and easy way to setthe optimum gate timings Fig. 5.

The FERA 4300 data are loaded to the CES RAM through a FERA 4301 controller. Once the data are loaded each CES module signals the Data acquisition computer that is ready for data transfer, by issuing an interrupt on the VME bus. The target proces ior then stores the data in its local RAM. The read out from $t: 3$ two CES modules takes one millisecond to complete. In oraer to acquire data at a faster rate (i.e. $10 \mathrm{kHz}$ ) during bursts, the readout is suspended and the data for each burst pulse are stacked in the CES RAM. Each CES is allowed to signal the VME for data transfer after the burst is completed.

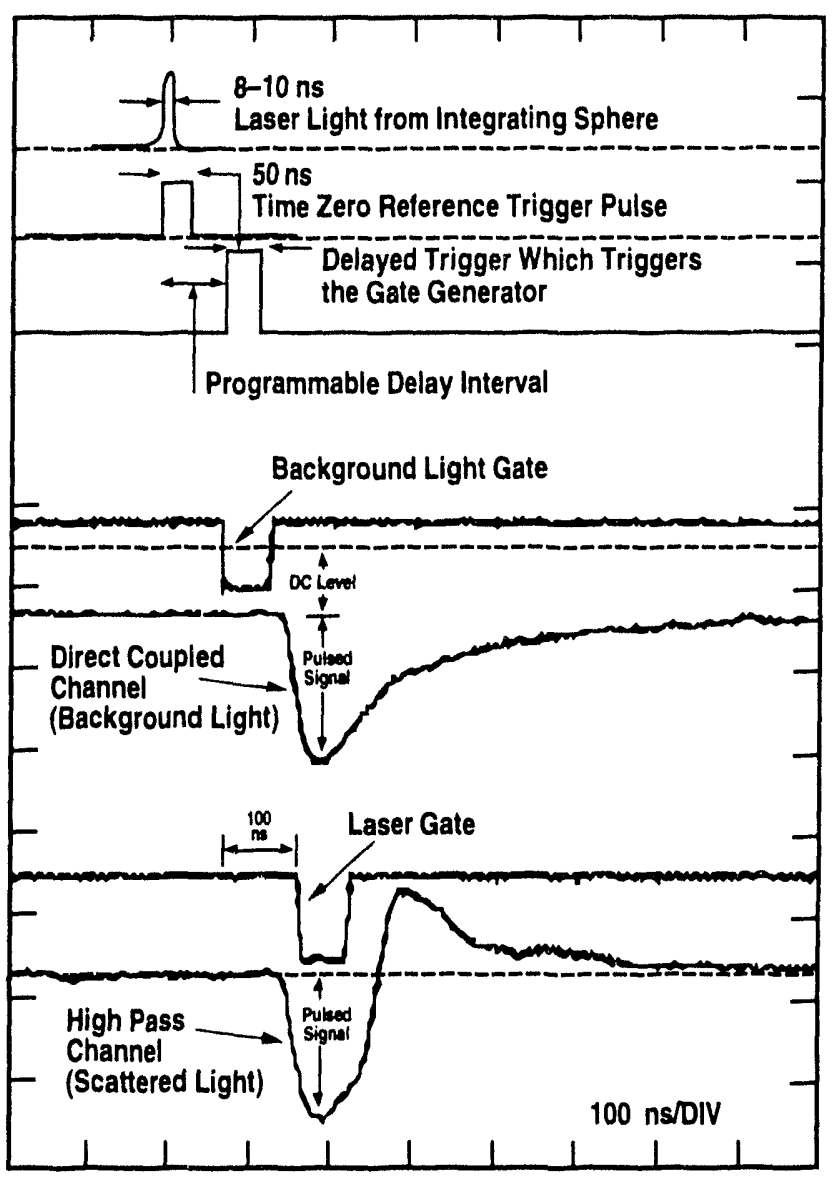

Fig. 5. Data acquisition timing.

CONCLUSION

An effective new laser control system has been built for the DIII-D Thomson Scattering system. The hardware control scheme has provided the ability to fire the lasers with $\pm 1 \mu \mathrm{s}$ timing accuracy. It has also provided $10 \mathrm{kHz}$ burst mode capability and has improved reliability. The modular VME format design provides flexibility to add new circuits and functionality to suit the needs of the experimenters. The combined laser firing capability will help in probing much lower plasma densities.

\section{REFERENCES}

[1] C.M. Greenfield, G.L. Campbell, T.N. Carlstrom, J.C. DeBoo, C.-L. Hsieh, R.T.Snider, P.K.Trost, "A real time digital control, data acquisition and analysis system for the DIII-D multipulse thomson scattering diagnostic," General Atomics Report GA-A20097.

[2] T.N. Carlstrom, G.L. Campbell, J.C. DeBoo, R. Evanko, J. Evans, C.M. Greenfield, et al... "Design and operation of the multipulse Thomson scattering diagnostic on DIII-D," Rev. Sci. Instrum. 63, pp. $4901-4906,1992$. 


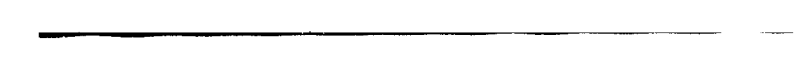

$\overline{-}-$

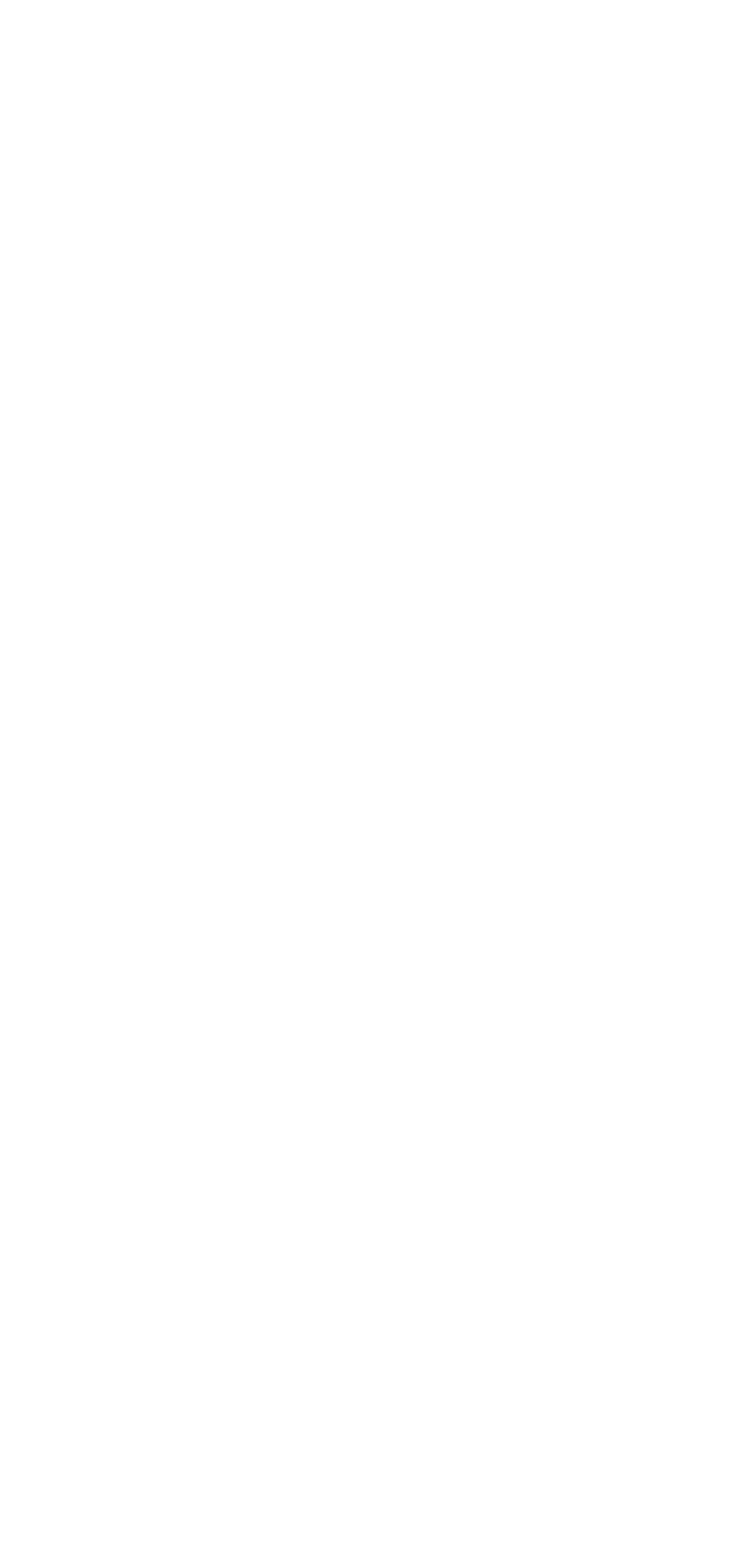

